\title{
EVLP CMV gB-pp65 Plus GM-CSF VBI-1901
}

National Cancer Institute

\section{Source}

National Cancer Institute. eVLP CMV gB-pp65 Plus GM-CSF VBI-1901. NCI Thesaurus. Code C143956.

A cancer vaccine composed of enveloped virus-like particles (eVLPs) containing the human cytomegalovirus (CMV) antigens glycoprotein B (gB) and phosphoprotein 65 (pp65; UL83) that are combined with the immunoadjuvant granulocyte-macrophage colony-stimulating factor (GM-CSF), with potential immunostimulatory and antineoplastic activities. Upon intradermal administration of VBI-1901, the eVLPs are taken up by and activate immune cells, such as dendritic cells (DCs), thereby stimulating the immune system to exert a cytotoxic $\mathrm{T}$-lymphocyte (CTL) immune response against CMV gB and pp65-expressing tumor cells. This may lyse and inhibit the proliferation of CMV-infected cancer cells. eVLPs, derived from viral structural proteins, stimulate the immune system and promote CTL responses. CMV gB and pp65 are expressed in certain tumor cell types, such as glioblastoma multiforme (GBM). GM-CSF stimulates the immune system and potentiates the antitumor immune response. 